\title{
A universal matter-wave interferometer with optical ionization gratings in the time domain
}

\author{
Philipp Haslinger, Nadine Dörre, Philipp Geyer, Jonas Rodewald, Stefan Nimmrichter \\ and Markus Arndt*
}

\begin{abstract}
Matter-wave interferometry with atoms ${ }^{1}$ and molecules ${ }^{2}$ has attracted a rapidly growing level of interest over the past two decades, both in demonstrations of fundamental quantum phenomena and in quantum-enhanced precision measurements. Such experiments exploit the non-classical superposition of two or more position and momentum states that are coherently split and rejoined to interfere ${ }^{3-11}$. Here, we present the experimental realization of a universal nearfield interferometer built from three short-pulse single-photon ionization gratings ${ }^{12,13}$. We observe quantum interference of fast molecular clusters, with a composite de Broglie wavelength as small as $\mathbf{2 7 5} \mathbf{f m}$. Optical ionization gratings are largely independent of the specific internal level structure and are therefore universally applicable to different kinds of nanoparticle, ranging from atoms to clusters, molecules and nanospheres. The interferometer is sensitive to fringe shifts as small as a few nanometres and yet robust against velocitydependent phase shifts, because the gratings exist only for nanoseconds and form an interferometer in the time domain.

Recent progress in atom interferometry has been driven by the development of wide-angle beam splitters ${ }^{14}$, large interferometer areas ${ }^{15}$ and long coherence times ${ }^{16}$. Most interferometers operate in a Mach-Zehnder ${ }^{5,17}$, Ramsey-Bordé ${ }^{18}$ or Talbot-Lau ${ }^{19}$ configuration, some of them also in the time domain ${ }^{20,21}$. Here we ask how to generalize these achievements to atoms, molecules, clusters or nanoparticles-irrespective of their internal states.

Mechanical nanomasks ${ }^{22}$ could be considered as universal if it were not for their van der Waals attraction on the traversing matter waves, which induces sizable dispersive, that is, velocity-dependent, phase shifts even for gratings as thin as $10 \mathrm{~nm}$.
\end{abstract}

Optical $^{9,14}$ or measurement-induced ${ }^{23}$ gratings eliminate this effect, but most methods so far relied on closed transitions and required an individual light source for every specific kind of atom or molecule.

It is possible to circumvent this restriction by using the spatially periodic electric dipole potential in an off-resonant standing light wave. Its field then modulates the phase of the matter wave rather than the amplitude. This implies, however, that the spatial coherence of the incident matter wave needs to be prepared by other means before-such as by collimation, cooling ${ }^{24}$ or the addition of another absorptive (material) mask ${ }^{2}$.

Here, we demonstrate a new method for coherence experiments with a wide class of massive particles and show how a sequence of ionizing laser grating pulses ${ }^{12}$ can form a generic matter-wave interferometer in the time domain ${ }^{13}$.

Figure 1 shows a schematic of the layout of our experiment, which we here realize specifically for clusters of anthracene (Ac) molecules. The molecules are evaporated in an Even-Lavie valve ${ }^{25}$ that injects the organic vapour with a pulse width of about $30 \mu \mathrm{s}$ into the vacuum chamber. The adiabatic co-expansion with a noble gas cools the molecules and fosters the formation of organic clusters - here typically up to $\mathrm{Ac}_{15}$.

The bunch of neutral nanoparticles passes a differential pumping stage, enters the interferometer chamber and flies in a short distance $(0.1-4 \mathrm{~mm})$ from the surface of a super-polished $\mathrm{CaF}_{2}$ mirror before it reaches the laser ionization region of a time-of-flight mass spectrometer (TOF-MS) where it creates the signal peaks.

The pulsed beams of three synchronized $\mathrm{F}_{2}$-excimer lasers $(\lambda=157.63 \mathrm{~nm})$ hit the mirror surface and the cluster beam under normal incidence with a variable pulse energy of $1-3 \mathrm{~mJ}$ and a duration of about $7 \mathrm{~ns}$. The laser beams are separated in space by $\sim 20 \mathrm{~mm}$ along the cluster trajectory. Their mutual time delay is adjusted with an accuracy of a few nanoseconds. We choose the laser beam diameters $(\sim 1 \mathrm{~mm} \times 10 \mathrm{~mm}$ rectangular flat top, extended along the cluster beam) to cover a wide particle bunch emitted by the source, whereas the detection laser beam is narrow enough to post-select only those clusters that have interacted with all three laser light pulses.

All three laser gratings interact with the matter waves in two different ways ${ }^{13}$ : they imprint a periodic phase and, more importantly, they act as transmission gratings because the photon energy of $\sim 7.9 \mathrm{eV}$ exceeds the ionization energy of the nanoclusters. Particles that traverse the antinodes of a laser grating ionize with high probability after absorption of one or more photons and a weak electric field removes them from the beam. Close to the nodes of the standing light waves the clusters remain neutral and move on in the interferometer. This process imprints a periodic modulation onto the matter-wave amplitude-as if the clusters had passed a mechanical nanomask.

A strong spatial localization inside the first laser grating is important for preparing a comb of emergent wavelets whose transverse coherence will cover a few antinodes in the second light grating further downstream. This is a prerequisite for interference to occur, that is, for the formation of a free-flying cluster density pattern at precisely defined moments in time, which is probed with nanosecond precision by the third ionizing standing wave.

The three laser pulses form a Talbot-Lau interferometer in the time domain, which exhibits transmission resonances when the delay between two subsequent pulses is close to the Talbot time $T_{m}=m d^{2} / h$, where $m$ is the cluster mass and $h$ is Planck's constant. In our setting, the grating period $d=\lambda / 2=78.8 \mathrm{~nm}$ results in $T_{m}=15 \mathrm{~ns} \mathrm{AMU}^{-1}$. All particles see the same gratings at the same time irrespective of their velocity. Even though they may enclose different areas in real space $(x-z)$, they will accumulate the same phase and contribute constructively to the same interferogram for each given mass (Fig. 1b). 

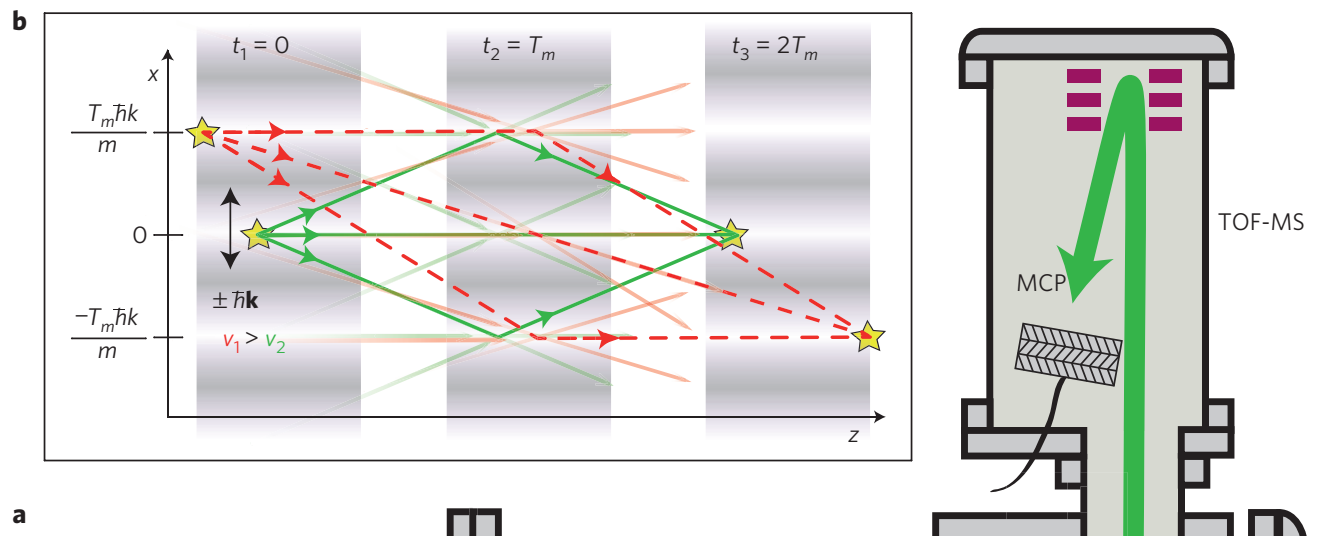

a

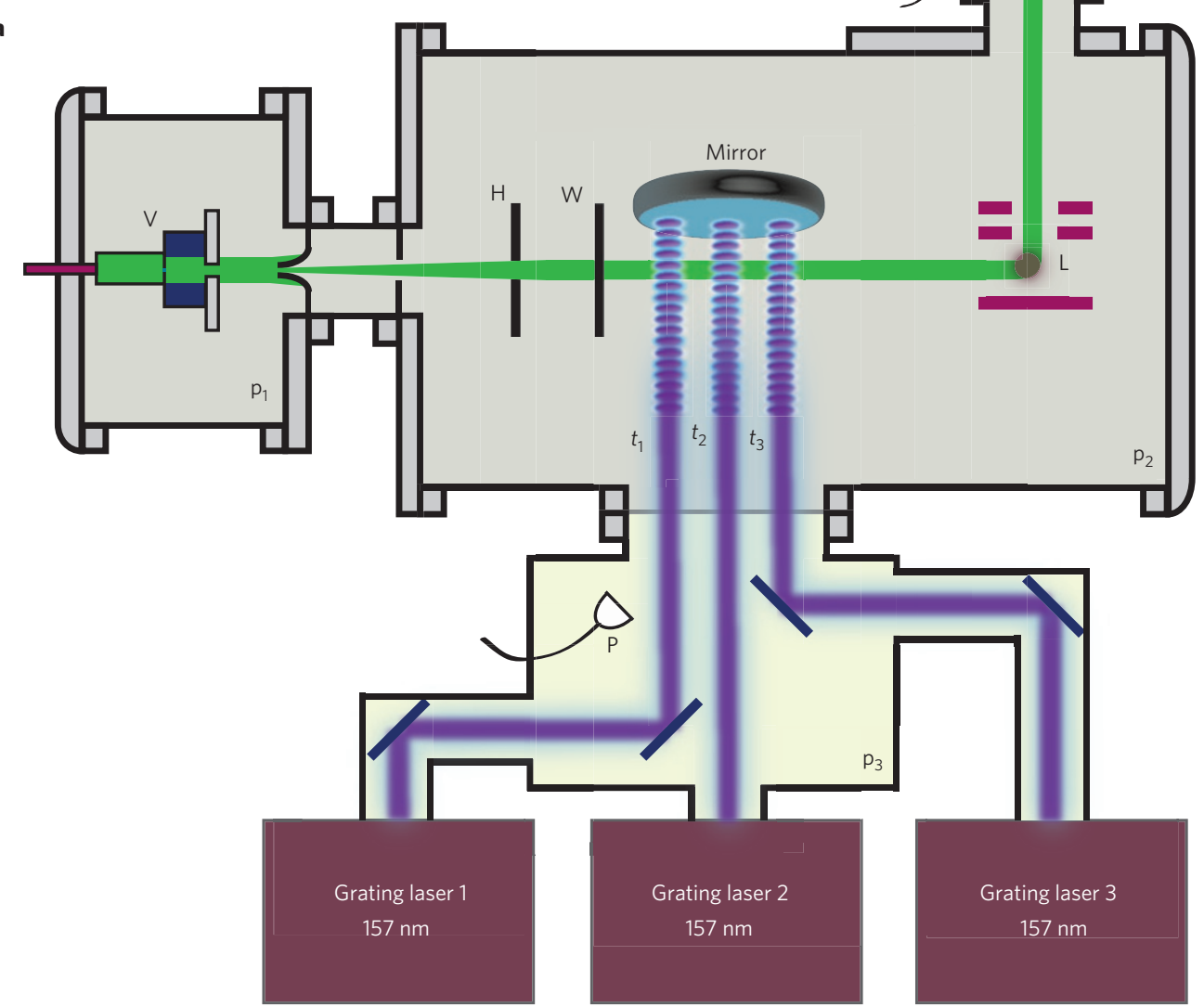

Figure 1 | Layout of the OTIMA interferometer. a, Set-up for nanoparticle interferometry with three short-pulse optical ionization gratings. From left to right: the Even-Lavie valve (V) produces a $30 \mu$ s pulse of neutral Ac clusters that are cooled in an adiabatic co-expansion with a noble gas jet. The cluster beam is delimited by two slits that are variable in height $(\mathrm{H})$ and width $(\mathrm{W})$. The laser pulses at $t_{1}=0, t_{2}=T$ and $t_{3}=2 T$ are back-reflected by a single 2-inch mirror to form three standing light waves. These are responsible for preparing the initial spatial coherence, for matter-wave diffraction and for spatially filtering the emerging cluster interferogram. The detection laser $(L)$ ionizes the transmitted neutral clusters for TOF-MS. A photodiode ( $P$ ) is used to monitor the laser timing with nanosecond accuracy. MCP, micro-channel plate. $\mathbf{b}$, The interferogram is formed by multiple paths from the first to the third grating that correspond to an effective momentum transfer of $n \hbar k$ in each grating, with $n \in Z$. Accurate timing ensures that the interfering paths branch and close at the same points on the grating axis $x$, irrespective of the cluster's initial velocities $v_{1}\left(\right.$ red) $>v_{2}$ (green). The stars indicate the localization of the matter waves.

We trace the emergent interference pattern in four different ways: its mass characteristics, its dependence on the pulse separation and pulse sequence asymmetry, and by visualizing its structure in position space.

We start by monitoring the TOF-MS signal and toggle between a resonant and a non-resonant setting. In the resonant mode the delays $t_{2}-t_{1}=T, t_{3}-t_{2}=T+\Delta T$ between two subsequent laser pulses are equal, $\Delta T=0$, and quantum interference is expected to modulate (enhance or reduce, depending on the phase) the transmission for the mass whose Talbot time matches the pulse separation $T$. In the off-resonant mode, the pulse delays are imbalanced by $\Delta T=200 \mathrm{~ns}$ and this tiny mismatch suffices to destroy the interferometric signal. We extract the interference contrast from the normalized difference $\Delta S_{\mathrm{N}}=\left(S_{\mathrm{R}}-S_{\mathrm{O}}\right) / S_{\mathrm{O}}$ between the resonant signal $S_{\mathrm{R}}$ and the off-resonant signal $S_{\mathrm{O}}$ and plot it as a function of mass in Fig. 2. The experimental mass spectra and $\Delta S_{\mathrm{N}}$ bars (green) can be well understood by a quantum mechanical model (violet bars), as described in Methods, and both are in marked discrepancy with a classical model (grey bars) ${ }^{13}$.

The role of the pulse separation $T$ is demonstrated by changing the seed gas from argon to neon. Shifting the most probable jet velocity from 690 to $925 \mathrm{~m} \mathrm{~s}^{-1}$ allows us to decrease $T$. The quantum model then predicts the highest contrast to occur at smaller masses, as confirmed by the experimental data in Fig. 2 b. 
a

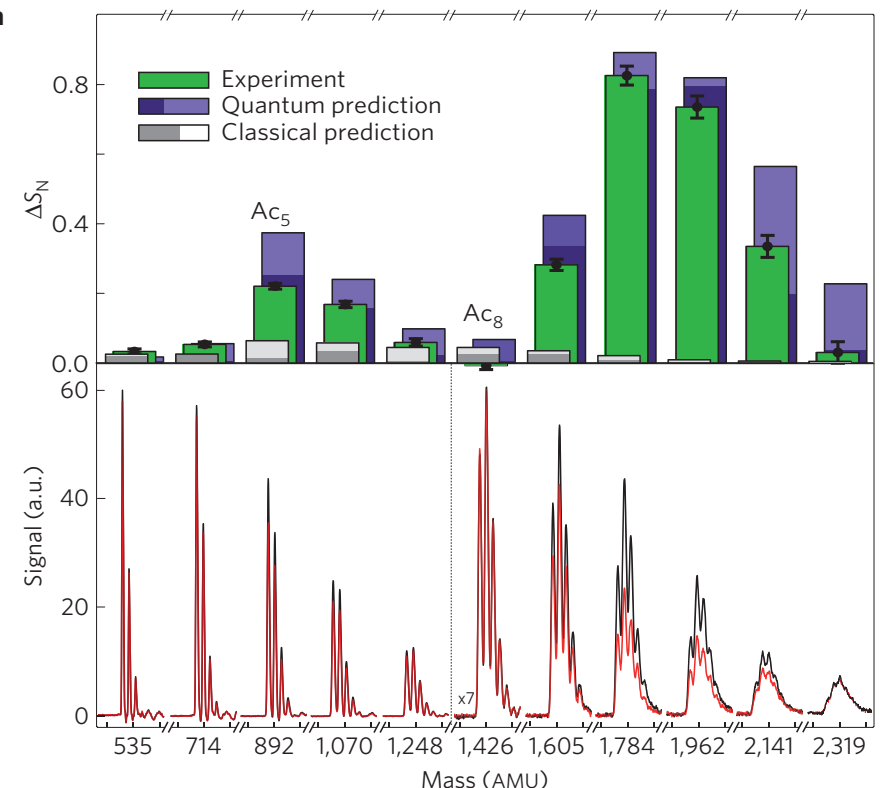

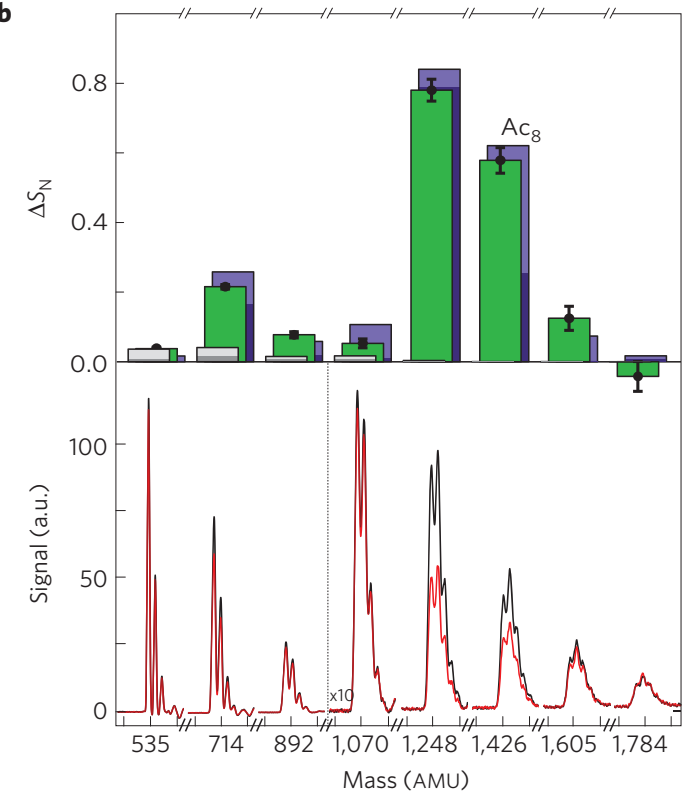

Figure 2 | Cluster interference visualized by means of the mass spectrum, for two pulse separation times. a, Lower panel: mass spectra recorded for a resonant (black line) and off-resonant ( $\Delta T=200 \mathrm{~ns}$, red line) pulse separation of $T=25.9 \mu$ s (clusters seeded in an argon jet). Each cluster signal splits into isotopic sub-peaks. The $x$-ticks correspond to a mass separation of $4 \mathrm{AMU}$. The two spectra differ for masses that fulfil $T_{m} \simeq T$. Upper panel: histogram of the cluster interference contrast, as measured by the signal difference $\Delta S_{N}$ integrated over the main isotopes of a given cluster. The predictions of the quantum/classical model $l^{13}$ are shown in violet/grey. The light violet/grey regions indicate the variation of the fringe contrast with a $\pm 30 \%$ variation of the cluster polarizability $\alpha_{157}$. For further details, see Methods and Supplementary Information Se. $\mathbf{b}$, The same as in a but with neon seeding and $T=18.9 \mu$ s. The error bars represent 1 s.d. of statistical error (see Supplementary Information Sh).

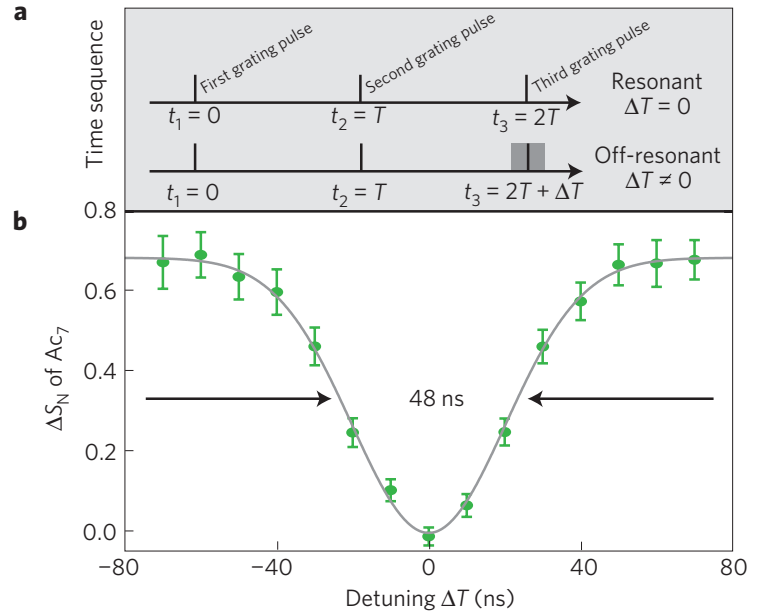

Figure 3 | Interferometric resonance and timing precision. Cluster self-imaging in a pulsed near-field interferometer is a resonant process with a short acceptance window for the matter waves to rephase. a, Pulse sequence. $\mathbf{b}$, Difference $\Delta S_{N}$ between the resonant and off-resonant signals detected at a mass of $A c_{7}$ as a function of $\Delta T$. In our set-up and for a pulse separation time $T$ of $18.9 \mu$ s, interference occurs during a time window of $48 \mathrm{~ns}$ (full-width at half-maximum). The error bars represent 1. s.d. of statistical error (see Supplementary Information Sh).

Figure 3 shows a clear resonance in $\Delta S_{\mathrm{N}}$ as a function of the time imbalance $\Delta T \in[-70,+70]$ ns with a width determined by the transverse momentum distribution of the cluster beam ${ }^{13}$. The momentum spread inferred from a Gaussian fit to the data in Fig. 3 corresponds to a divergence angle along the grating of $2.1 \mathrm{mrad}$, in good agreement with the experimental settings.

In our set-up, the pulsed supersonic expansion determines the cluster velocity distribution and the pulsed mass detection post-selects its relative width to $\Delta v / v \simeq 3 \%$. It is then justified to interpret the observations in position space: with the de Broglie wavelength given by $\lambda_{\mathrm{dB}}=h / m v$, the mass distribution also represents a wavelength spectrum. The most prominent interference peak in Fig. $2 \mathrm{~b}$ at 1,248 AMU corresponds to the heptamer $\mathrm{Ac}_{7}$ with $\lambda_{\mathrm{dB}} \simeq 345 \mathrm{fm}$, at $v \simeq 925 \mathrm{~m} \mathrm{~s}^{-1}$. The highest mass peaks in the spectrum reach down to below $\lambda_{\mathrm{dB}} \simeq 275 \mathrm{fm}$.

Finally, we can also prove the formation of an interference pattern in real space by modifying the period of the central grating: whereas all laser beams had originally been set to normal incidence on the interferometer mirror-with an uncertainty of about $200 \mu \mathrm{rad}$-we now explicitly tilt the central laser beam by $5.1 \mathrm{mrad}$ along the cluster beam. The direction of the standinglight-wave grating remains defined by the orientation of the mirror surface, but an increasing tilt angle $\theta$ reduces the modulus of the wave vector perpendicular to the surface, $k_{\mathrm{p}}=k \cdot \cos \theta$. We can shift the interference pattern by half a grating period when the clusters pass the mirror surface at an average distance of $1.5 \mathrm{~mm}$. We plot the fringe shift as a function of the separation between the beam and the mirror in Fig. 4 and find a damped sinusoidal transmission curve for all clusters with the expected period. The overall damping results from the limited coherence of the laser system and the vertical extension of the Ac cluster beam.

All tests presented here confirm the successful experimental realization of an optical time-domain ionizing matter-wave (OTIMA) interferometer ${ }^{2,13}$, which exploits pulsed ionization gratings. This versatile tool for quantum interferometry will be applicable to a large class of nanoparticles.

Owing to the pulsed gratings, all phase shifts caused by constant external forces become velocity-independent and leave the contrast unaffected. The dispersive Coriolis shift ${ }^{15}$ can be well compensated by a suitable orientation of the interferometer, if needed.

The wide applicability and non-dispersive nature of pulsed ionization gratings make the OTIMA design particularly appealing for quantum experiments with highly complex particles, eventually 


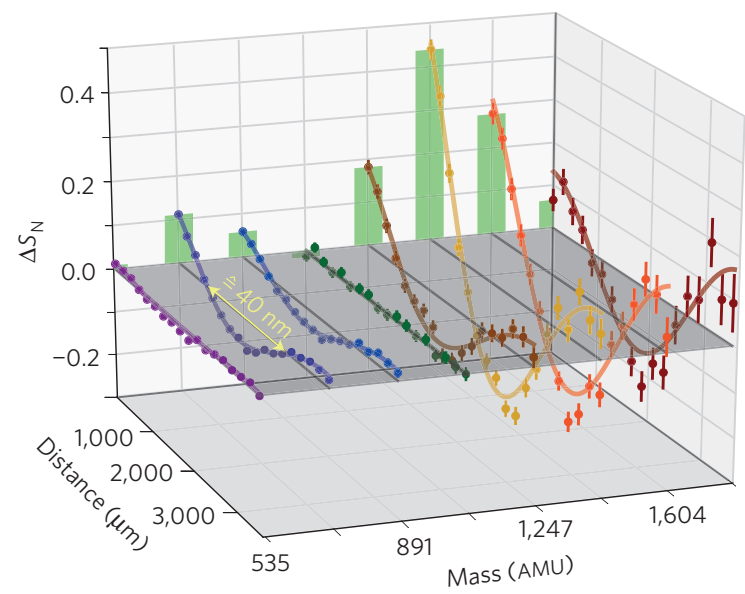

Figure $4 \mid \Delta S_{N}$ as a function of the mirror displacement for different clusters. The second grating laser beam was tilted by $5.1 \pm 0.3 \mathrm{mrad}$ in the direction of the molecular beam to stretch the effective grating period by about 0.013 per mille. This suffices to induce a fringe shift of half a grating period for molecules travelling around $1.5 \mathrm{~mm}$ distance from the mirror surface. The mirror height is varied to effectively shift the second grating with regard to the other two, which allows us to scan the cluster interference pattern. We extract the periodicity for $\Delta S_{N}$ as a function of the mirror distance by fitting a damped sine curve to the experimental data. This periodicity corresponds to the expected effective period ${ }^{13}$ of the interferogram of about $78.8 \mathrm{~nm}$. The error bars represent $1 \mathrm{~s}$.d. of statistical error (see Supplementary Information Sh).

even with nanoparticles at the length scale of the grating period. As high-mass interferometry requires coherence of the order of the Talbot time, practical mass limits are imposed by free fall in the gravitational field on Earth in combination with the limited coherence of vacuum ultraviolet lasers and the finite phase-space density of the available particle sources. However, none of them is fundamental. Even in the presence of thermal radiation at room temperature (particle and environment) and collisional decoherence at a background pressure of $10^{-9} \mathrm{mbar}$, the OTIMA design is predicted to enable new tests of quantum physics, such as tests of spontaneous localization, with particle masses around $10^{6}$ AMU and beyond ${ }^{26}$.

On the applied side, the OTIMA set-up is expected to improve the accuracy of molecule and cluster deflectometry because it ensures the same interaction (phase accumulation) time for all particles with the external fields ${ }^{27}$ and a position readout at the nanometre scale. Our interferometer concept therefore establishes also the basis for a new class of quantum-enhanced precision metrology experiments.

\footnotetext{
Methods

Absorption and optical polarizability. The central grating influences the propagation of the coherent matter wave by modulating both its amplitude and phase. It does this by removing particles from the anti-nodes of the standing light field and by imprinting a phase onto the matter wave in proportion to the clusters' optical polarizability at $157 \mathrm{~nm}$. In the first and third grating the phase modulation has no effect, because the clusters enter with random phases, and because the last grating merely acts as a transmission mask. Neither the absorption cross-sections $\sigma_{157}(N)$ nor the polarizabilities $\alpha_{157}(N)$ are known, a priori, for each cluster of $N$ molecules in the vacuum ultraviolet wavelength range. However, $\sigma_{157}(N)$ enters the model only through the mean number of photons absorbed $n_{0}(N)$ in each grating, which we can determine by monitoring the cluster loss rate. Whereas this parameter influences the general shape of the interference curve as a function of mass, the polarizability may modify the predicted contrast of each individual cluster. We assume the polarizability and the absorption cross-section to exhibit the same $\mathrm{N}$-scaling as retrieved from our $n_{0}(N)$ measurements and we allow the polarizability to vary by $\pm 30 \%$ (light violet confidence areas in Fig. 2) around the single-molecule value. We use the polarizability
}

$\alpha_{157}(1)=25.4 \times 10^{-30} \mathrm{~m}^{3}$ from ref. 28 and we extract an absorption cross-section of $\sigma_{157}(1)=1.1 \times 10^{-20} \mathrm{~m}^{2}$ from ref. 29 .

This yields the quantum and classical theory curve in Fig. 2. Apart from the uncertain polarizability, the deviations from the experimental data may be attributed to a limited efficiency of single-photon ionization and contributions by fragmentation processes. Although the absolute interference contrast is sensitive to a variety of different cluster properties that are still to be extracted in combination with more refined cluster theory, the fringe shift will become valuable for precisely measuring the interplay between internal cluster properties and external forces.

Received 2 November 2012; accepted 24 December 2012; published online 10 February 2013

\section{References}

1. Cronin, A. D., Schmiedmayer, J. \& Pritchard, D. E. Optics and interferometry with atoms and molecules. Rev. Mod. Phys. 81, 1051-1129 (2009).

2. Hornberger, K., Gerlich, S., Haslinger, P., Nimmrichter, S. \& Arndt, M. Colloquium: Quantum interference of clusters and molecules. Rev. Mod. Phys. 84, 157-173 (2012).

3. Estermann, I. \& Stern, O. Beugung von Molekularstrahlen. Z. Phys. 61, 95-125 (1930).

4. Keith, D. W., Schattenburg, M. L., Smith, H. I. \& Pritchard, D. E. Diffraction of atoms by a transmission grating. Phys. Rev. Lett. 61, 1580-1583 (1988).

5. Kasevich, M. \& Chu, S. Atomic interferometry using stimulated Raman transitions. Phys. Rev. Lett. 67, 181-184 (1991).

6. Bordé, C. Atomic interferometry with internal state labelling. Phys. Lett. A 140, 10 (1989).

7. Rasel, E. M., Oberthaler, M. K., Batelaan, H., Schmiedmayer, J. \& Zeilinger, A. Atom wave interferometry with diffraction gratings of light. Phys. Rev. Lett. 75, 2633-2637 (1995).

8. Fray, S., Diez, C., Hänsch, T. \& Weitz, M. Atomic interferometer with amplitude gratings of light and its applications to atom based tests of the equivalence principle. Phys. Rev. Lett. 93, 240404 (2004).

9. Müller, H., Chiow, S-w., Long, Q., Herrmann, S. \& Chu, S. Atom interferometry with up to 24-photon-momentum-transfer beam splitters. Phys. Rev. Lett. 100, 180405 (2008).

10. Moskowitz, P. E., Gould, P. L., Atlas, S. R. \& Pritchard, D. E. Diffraction of an atomic beam by standing-wave radiation. Phys. Rev. Lett. 51, 370-373 (1983).

11. Giltner, D. M., McGowan, R. W. \& Lee, S. A. Atom interferometer based on Bragg scattering from standing light waves. Phys. Rev. Lett. 75, 2638-2641 (1995).

12. Reiger, E., Hackermüller, L., Berninger, M. \& Arndt, M. Exploration of gold nanoparticle beams for matter wave interferometry. Opt. Commun. 264, 326-332 (2006).

13. Nimmrichter, S., Haslinger, P., Hornberger, K. \& Arndt, M. Concept of an ionizing time-domain matter-wave interferometer. New. J. Phys. 13, 075002 (2011).

14. Chiow, S., Kovachy, T., Chien, H. C. \& Kasevich, M. A. $102 \hbar k$ large area atom interferometers. Phys. Rev. Lett. 107, 130403 (2011).

15. Lan, S-Y., Kuan, P-C., Estey, B., Haslinger, P. \& Müller, H. Influence of the coriolis force in atom interferometry. Phys. Rev. Lett. 108, 090402 (2012).

16. Müller, H., Chiow, S., Herrmann, S., Chu, S. \& Chung, K. Y. Atom-interferometry tests of the isotropy of post-Newtonian gravity. Phys. Rev. Lett. 100, 31101 (2008).

17. Keith, D. W., Ekstrom, C. R., Turchette, Q. A. \& Pritchard, D. E. An interferometer for atoms. Phys. Rev. Lett. 66, 2693 (1991).

18. Bordé, C. J., Courtier, N., Burck, F. D., Goncharov, A. N. \& Gorlicki, M. Molecular interferometry experiments. Phys. Lett. A 188, 187-197 (1994).

19. Clauser, J. F. \& Li, S. Talbot-von Lau atom interferometry with cold slow potassium. Phys. Rev. A 49, R2213 (1994).

20. Szriftgiser, P., Guéry-Odelin, D., Arndt, M. \& Dalibard, J. Atomic wave diffraction and interference using temporal slits. Phys. Rev. Lett. 77, 4-7 (1996).

21. Cahn, S. B. et al. Time-domain de Broglie wave interferometry. Phys. Rev. Lett. 79, 784-787 (1997).

22. Juffmann, T. et al. Real-time single-molecule imaging of quantum interference. Nature Nanotech. 7, 297-300 (2012).

23. Storey, P., Collett, M. \& Walls, D. Measurement-induced diffraction and interference of atoms. Phys. Rev. Lett. 68, 472-475 (1992).

24. Deng, L. et al. Temporal, matter-wave-dispersion Talbot effect. Phys. Rev. Lett. 83, 5407-5411 (1999).

25. Even, U., Jortner, J., Noy, D., Lavie, N. \& Cossart-Magos, C. Cooling of large molecules below $1 \mathrm{~K}$ and He clusters formation. J. Chem. Phys. 112, 8068-8071 (2000).

26. Nimmrichter, S., Hornberger, K., Haslinger, P. \& Arndt, M. Testing spontaneous localization theories with matter-wave interferometry. Phys. Rev. A 83, 043621 (2011). 
27. De Heer, W. A. \& Kresin, V. V. in Handbook of Nanophysics: Clusters and Fullerenes (ed. Sattler, K. D.) Ch. 10 (CRC Press, 2011).

28. Marchese, F. T. \& Jaffé, H. H. The calculation of ground and excited state molecular polarizabilities: A simple perturbation treatment. Theor. Chim. Acto 45, 241-247 (1977).

29. Malloci, G., Mulas, G. \& Joblin, C. Electronic absorption spectra of PAHs up to vacuum UV Towards a detailed model of interstellar PAH photophysics. Astron. Astrophys. 117, 105-117 (2004).

\section{Acknowledgements}

We acknowledge support by the Austrian science funds (FWF-Z149-N16 Wittgenstein and DK CoQuS W1210-2) as well as infrastructure funds by the Austrian ministry of science and research BMWF (IS725001). We thank U. Even and O. Cheshnovsky for emphasizing the benefits of organic molecules with the Even-Lavie valve and $\mathrm{K}$. Hornberger for collaborations on the modelling of the OTIMA interferometer. We thank B. von Issendorff for discussions on cluster sources.

\section{Author contributions}

P.H., N.D., P.G. and J.R. built the interferometer and performed the measurements. P.H. contributed the initial experimental layout and the time-domain perspective of the experiment. P.G. developed the customized software and data acquisition system in feedback with the team. Data analysis was done by N.D., J.R. and P.H. S.N. contributed the theoretical description. M.A. initiated and supervised the experiment. P.H., N.D., J.R., S.N. and M.A. wrote the paper with input by all co-authors.

\section{Additional information}

Supplementary information is available in the online version of the paper. Reprints and permissions information is available online at www.nature.com/reprints. Correspondence and requests for materials should be addressed to M.A.

\section{Competing financial interests}

The authors declare no competing financial interests. 